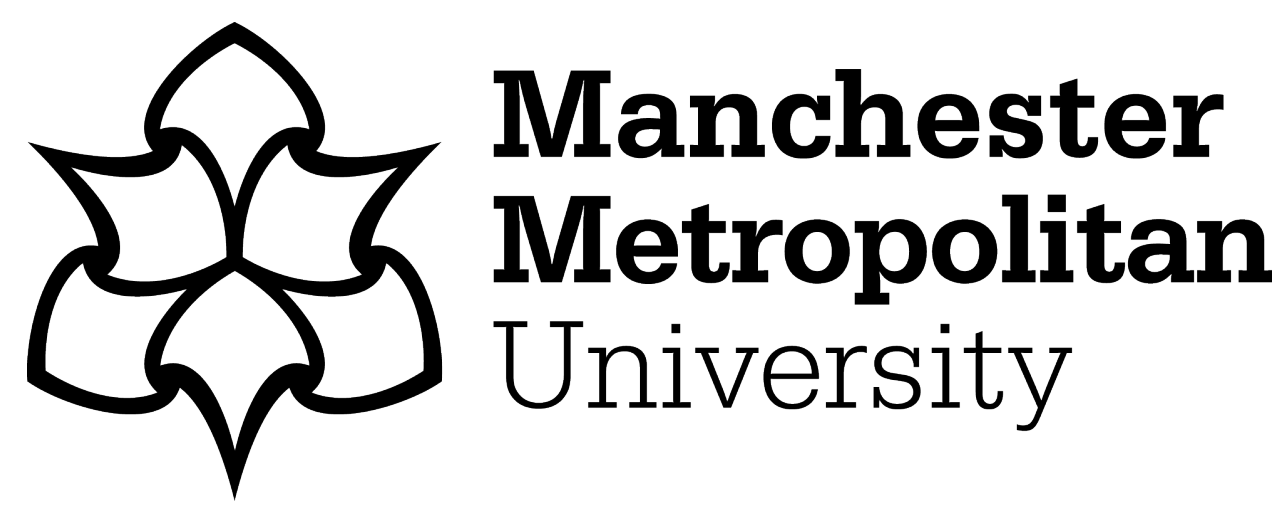

Carolan, K, Verran, J, Amos, M, Crossley, M, Redfern, J, Whitton, N and Louttit, D (2018) SimFection: a digital resource for vaccination education. Journal of Biological Education, 53 (2). pp. 225-234. ISSN 0021-9266

Downloaded from: https://e-space.mmu.ac.uk/620731/

Version: Accepted Version

Publisher: Taylor \& Francis (Routledge)

DOI: https://doi.org/10.1080/00219266.2018.1469534

Please cite the published version 


\section{SimFection: A Digital Educational Resource for Vaccination Education}

Carolan, K. ${ }^{a}$, Verran, J. ${ }^{b^{*}}$, Amos, M. ${ }^{\mathrm{a}}$, Crossley, M. ${ }^{\mathrm{a}}$, Redfern, J. ${ }^{\mathrm{b}}$, Whitton, N. ${ }^{\mathrm{c}}$, Louttit, D. ${ }^{\mathrm{b}}$

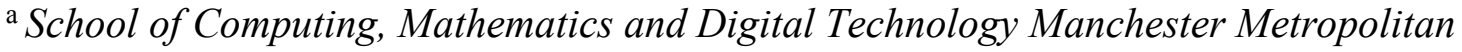
University, Chester St., UK;

${ }^{\mathrm{b}}$ School of Healthcare ScienceManchester Metropolitan University, Manchester, UK;

${ }^{\mathrm{c}}$ Faculty of Education, Manchester Metropolitan University, Manchester, UK

Corresponding author: Joanna Verran

Email: j.verrran@mmu.ac.uk

00441612471206 


\title{
SimFection: A Digital Educational Resource for Vaccination Education
}

\author{
Abstract \\ Vaccination coverage in the United Kingdom is below the level recommended by the \\ World Health Organisation, and when vaccination coverage is not sufficient, outbreaks \\ of infectious diseases can occur. In 2015, coverage of the first dose of the Measles- \\ Mumps-Rubella vaccine declined in the United Kingdom for the first time since 2008, \\ indicating a need to raise public awareness and understanding of the importance of \\ vaccination to public health. Identifying 16 -18-year olds as a target audience, being \\ future parents who would make decisions regarding vaccination of their children, a \\ digital educational resource ('SimFection') was developed to deliver key messages \\ about the spread and control of vaccine-preventable infectious diseases (identified via \\ school curricula). The process of development utilised an iterative approach, involving \\ a cyclic process of prototyping, testing, analysis and refinement with a range of \\ audiences including students, schoolchildren, and trainee teachers. The completed \\ resource is now available online for free download.
}

Keywords: vaccination; digital; educational; resource; infectious

\section{Introduction}

Public understanding of the spread of infectious disease is an important issue for the maintenance and improvement of public health (World Health Organisation 2012; https://www.gov.uk/government/organisations/health-and-social-care-information-centre). Vaccination coverage in the United Kingdom is below the level recommended by the World Health Organisation (World Health Organisation 2012), and when vaccination coverage is not sufficiently high, outbreaks of disease can occur (Jansen et al. 2003). Whilst vaccination coverage has improved since 1988, vaccine coverage of the first dose of the Measles-MumpsRubella vaccine (MMR1) declined in 2015 in the United Kingdom for the first time since 2008 (https://www.gov.uk/government/organisations/health-and-social-care-informationcentre 
Previous vaccination interventions aimed at adults have also had limited effectiveness, and can actually decrease intent to vaccinate (Nyhan et al. 2014; Dube, Gagnon, and MacDonald 2015). For this reason, the age group that would form an alternative target population for investigation was considered; the target group for this research focussed on the vaccination attitudes of teenagers. This group was selected for two main reasons: (1) They will be the next generation to make vaccination decisions about their own children, and (2) Teenagers have previously expressed interest in receiving more information about vaccination before making vaccination decisions (Gowda et al. 2012).

The use of digital resources in educational environments is increasing, with digital technology now an integral part of education (Selwyn and Cooper 2015). Thus, a digital educational resource entitled 'SimFection' was developed to deliver key messages about the spread of specific vaccine-preventable infectious diseases (identified via the school curricula) to A Level students (ages 16-18). 'SimFection' was based on computer simulation software developed by the authors (Crossley and Amos 2011) that was used to develop the game 'SimZombie' (Verran et al., 2014), which shows how outbreaks caused by zombies, werewolves and vampires spread through a population, and allows uninfected agents to 'fight back' against the monsters to prevent spread. SimZombie has been successfully used to engage a range of audiences at a variety of events including Cheltenham Science Festival (2014), Manchester Science Festival (2011 and 2012), Manchester Children's Book Festival (2010 and 2012) and 'Saturday Science' at the Museum of Science and Industry in Manchester (2013). Due to the observed success of SimZombie in engaging teenagers and families about the spread of infectious diseases (Verran et al., 2013), it was modified into SimFection, which modelled aspects of the epidemiology of selected infectious diseases, 
intended for a teenage audience, primarily delivered in school/college.

The aim of this paper is to document the development of SimFection as a robust educational tool for its target audience.

\section{Methods}

\section{Selection of Vaccine-preventable Diseases}

To identify those diseases that would best demonstrate key aspects of vaccination and disease transmission, a search was made of the current A Level Biology curriculum. Information about the spread of infectious diseases and vaccination is included in A Level specifications for AQA, OCR and WJEC (AQA 2014; OCR 2013; WJEC 2010).

The four diseases selected were caused by viruses: measles, mumps, influenza and smallpox. A particular message was identified for each virus, which could be demonstrated using SimFection. As with all vaccines, prevention of measles requires high vaccine coverage and good herd immunity, otherwise outbreaks can occur (Ramsay 2013). Mumps outbreaks can occur where a particular age group is insufficiently immunised, and moved to an area where this low coverage enabled spread (2005 outbreak of mumps in the UK [(Gupta, Best, and MacMahon 2005) and 2009 in UK universities [Kay et al. 2011]). Influenza vaccines must be continually modified as the virus undergoes antigenic drift and shift (Subbarao and Joseph 2007), and infectivity and virulence are different properties that may be demonstrated by different strains (for example, H1N1 (Virus and Team 2009) and H5N1 (Li et al. 2004)). Smallpox was declared eradicated in 1980, where the strategy of ring vaccination enabled contacts of cases to be immunised (Fenner 2011). 


\section{The Prototype SimFection Package}

SimZombie is an agent-based software simulation tool, programed using Java. Agent-based modelling is a computational method that enables a researcher to create, analyse, and experiment with models composed of agents that interact within an environment (Gilbert 2008).SimZombie simulates the spread of infection through an animated population of individuals, using an individual- based version of a standard susceptible/infected/recovered (SIR) model (Munz et al. 2009), and graphically depicts different categories of individual over time, using 'monster infections' (zombies, werewolves and vampires) rather than actual diseases. The SimZombie software was modified to address the epidemiology of the four viral infections.

Data for each infectious disease were collected (Table 1) and the information was used to pre-set the parameters used in SimFection. The parameters dictate the way in which each infectious disease spreads - for example, measles is the most infectious of the diseases used in SimFection and so in simulations it also spreads more rapidly than the other diseases. Each disease simulation was used to address a topic about vaccines and disease epidemiology:

The measles simulation allowed the percentage of individuals immunised in a population to be altered, and the impact of that change to be visualised (Figure 1a).

The smallpox simulation allowed users to alter the speed at which agents moved. The aim of the smallpox simulation was to use ring vaccination to prevent the spread of smallpox, thus increasing the speed at which agents move made it more difficult for users to prevent the spread of smallpox (Figure 1b).

The influenza simulation allowed users to alter infectivity and virulence of the influenza virus to investigate the effects of these changes. A strain with low infectivity and high virulence (such as H5N1 bird flu) would present a different simulation to a strain with high infectivity and low virulence (such as the $2009 \mathrm{H} 1 \mathrm{~N} 1$ pandemic) (Figure 1c). 
The mumps simulation allowed users to 'pick up' and move groups from one population with high vaccination coverage to one of low vaccination coverage (from low to high susceptibility) (Figure 1d).

The user interface was designed to be easy to use. The program included information about each infectious disease and instructions on how to run each simulation with a slider to change parameters as described above.

Within each simulation there are six classes of agents (individuals): immunised, carrying, susceptible, recovered, infected and dead. Each agent is represented by a dot coloured to indicate its class (for example immunised agents were coloured blue and infected agents coloured dark green). The agents move around the simulation randomly and encounter other agents, with outcomes based on input parameters. For example, if an infected agent encounters a susceptible agent, the susceptible may also become infected depending on how infectious the disease is.

Five PowerPoint presentations were developed for delivery alongside the simulation software. These included an introductory 'Viruses and Vaccination' presentation and a presentation for each of the four infectious diseases. These included background information, symptoms, treatment and prevention and a case study of an outbreak of each disease (for example the 2012 outbreak of measles in Swansea, Wales). In addition, a teacher's guide in pdf format was provided to assist teachers in using the learning package.

\section{Refining the Prototype}

There were several phases in the development of the SimFection learning package (Figure 2). The process of development was iterative involving a cyclic process of prototyping, testing, analysis and further refinement of a product. Iterative design is used in the development of games(Zimmerman 2014). At each stage, following feedback collected from focus groups and trials, improvements were made to both the software and accompanying materials. 
After initial development of the software, an informal but thorough focus group $(n=2)$ was held with undergraduate Biology students at the University. The participants were given the software and were asked for feedback. The participants were generally positive, but gave some useful suggestions, particularly for a quiz at the end of a session to test learning. Thus questions developed were based on the information provided by the PowerPoint presentations. The quiz requires two teams, the 'Infection team' and the 'Population team'. The aim for the 'Infection team' is to infect or kill the most people, whereas the aim for the 'Population team' is to prevent the spread of infectious disease to reduce deaths. Each question answered correctly by a team allows a certain parameter in the simulation to be changed, for example increasing the mortality rate or immunising 50 people. This changes the way in which the simulation progresses, allowing one team to win after a set number of rounds.

\section{Delivery to Target Group}

SimFection was delivered to the target group ( $\mathrm{n}=36$ students aged 16-18) in June 2014. The two-hour session began with a brief introduction to infectious diseases and SimFection, followed by use of the SimFection software. Each student had access to a laptop so could work through the activities individually. After exploring each infectious disease, the class was divided into two groups to complete the competitive quiz. The participants were largely attentive and participated in the sessions well, answering questions and engaging with group discussion. Feedback was collected by a 5-point Likert scale questionnaire and space was provided for written feedback (SupplementS1).

The feedback from the questionnaire was mostly positive and suggested that the software was informative, user-friendly and acceptable to the target group: 
- $100 \%(n=36)$ of the participants said they found SimFection informative.

- $89 \%(\mathrm{n}=32)$ said that that they had learnt something new.

- $77 \%(\mathrm{n}=28)$ said that SimFection was a good way to learn about infectious diseases.

- $61 \%(\mathrm{n}=22)$ said they found the software easy to use.

- $55 \%(\mathrm{n}=20)$ said they thought the software was well designed.

Following written feedback from the student participants, some improvements were made to the quiz including increasing the length of time provided to users to answer questions and more questions were added to the question bank as suggested by students and teaching staff observing the session. The ability to select sets of questions to answer was also added so that users could include or exclude certain questions based on which infectious diseases they had explored.

\section{Delivery to Trainee Teachers}

SimFection was delivered to trainee science teachers $(n=12)$ in January 2015 to gauge its potential educational value. This project targets teenagers and delivering the intervention in an educational setting will allow wide distribution of the intervention to the target group. For this reason, it was important to assess teachers' views on the educational content of the software and the likelihood of them using it.

Written feedback was collected by questionnaire (Supplement S2) and was somewhat positive. Their feedback was coded based on their responses to each question, for example, responses to the question 'Was the language used to be appropriate for GCSE and A Level students?' that were positive were coded 'positive', and negative responses were coded as 'negative': 
- 6 of the participants found the language used to be appropriate for GCSE and A Level students.

- 6 found that the content was suitable for GCSE and A Level Biology.

- 1 of the participants said they liked the design of the software.

- 3 said they would use the whole resource in their own teaching.

- 7 found the software user friendly.

- 7 found the instructions for use clear.

Space was also provided for participants to provide written feedback: suggestions and responses are presented in Table 4.

\section{Delivery of Modified Software to Trainee Teachers}

After the modifications (Supplement S3) had been made, the SimFection resource was delivered to a different cohort of trainee teachers $(n=24)$ in April 2015 to enable a second review. As with the previous cohort, the teachers were asked to work through the software and were provided with printed copies of the supporting documents, with the addition of the newly developed 'cheat sheet' (a shortened version of the full Teacher's Guide, providing basic information about the learning package) and extension activities. Feedback was collected using a questionnaire at the end of the session, using the same questionnaire used with the previous teacher group.

The feedback was mostly positive and teachers' responses were more favourable when compared with the first trial (Figure 3):

- $80 \%(\mathrm{n}=19)$ found the language suitable for KS4 and KS5 students.

- $\quad 87.5 \%(n=21)$ found the content suitable for GCSE and A Level Biology lessons.

- $25 \%(\mathrm{n}=6)$ liked the 'design' of the software (the way the software looks). 
- $\quad 92 \%(\mathrm{n}=22)$ said they would $u$ se the whole resource.

- $71 \%(\mathrm{n}=17)$ found the software user friendly.

- $67 \%(\mathrm{n}=16)$ found the instructions clear.

There was an increase in positive responses across all questions after modification of the resource, although the design of the software was not to everyone's taste. The more positive responses given for all questions after improvements were made to the resource (Figure 3), were not significantly better than previous responses.

Participants again made several suggestions for how the educational resource might be improved (Supplement S4). In addition to these changes, other changes included the addition of possible extension activities added to the 'Teachers' Guide' such as suggested research project topics, and the inclusion of all information from the PowerPoint presentations within the software itself. This would allow the software to be used as a 'standalone' educational resource, allowing versatility for use (teachers may lead a class and use elements of the software in front of the class, or students could be allowed to work through the software on their own or in pairs with less input from the teacher).

\section{Delivery of Modified Software to Target Group}

The software was delivered to students from a local school ( $\mathrm{n}=20$,age 16) in December 2015. The session began with a brief introduction and then students were asked to work individually through the activities contained in the software. Feedback was collected using the same questionnaire used by the previous group of students trialling the software.

The majority for feedback collected was positive: 
- $100 \%(\mathrm{n}=20)$ found the session informative

- $\quad 95 \%(\mathrm{n}=19)$ found the session interesting

- $60 \%(\mathrm{n}=12)$ found the software 'fun'

- $\quad 90 \%(\mathrm{n}=18)$ said they learnt something new from SimFection

- $85 \%(\mathrm{n}=17)$ felt that SimFection is a good way to learn about infectious diseases

- $85 \%(\mathrm{n}=17)$ found the software easy to use

- $85 \%(\mathrm{n}=17)$ said that the software was well designed

The feedback showed an increase in the number of positive responses to the questionnaire when compared to the first trial held with students (Figure 4). This may be due to the improvements made to the software since the first trial. More students found the software easy to use and thought it well-designed when compared with the first trial with students. However, whilst levels of agreement with each statement generally increased (Figure 4), only responses to question 2 'The workshop was interesting' $(\mathrm{p}=0.37, \mathrm{df}=1)$, question 6 'I found the resource easy to use' $(\mathrm{p}=0.28, \mathrm{df}=1)$ and 7 'SimFection is well designed' $(\mathrm{p}=0.016, \mathrm{~d}=1)$ were significantly higher after improvements were made.

\section{The Complete Resource}

A focus group ( $\mathrm{n}=5$ ) was held with postgraduate students and staff at the university to explore the resource, and to identify any final changes that might be required. Feedback suggested simplification of instructions for the mumps and smallpox activities, minor typographical changes, and the addition of general instructions for use of the software within the software. The final resource was made available on www.simfection.org.uk. 


\section{Conclusion}

The aim of this study was to develop an educational resource that demonstrated the spread of infectious disease and the role of vaccination in its prevention. Prototype packages were evaluated by a number of audiences, to ensure that the final package was robust and relevant. The iterative approach, although time-consuming, proved to be good practice in the development of this educational resource; refinement of the learning package received more positive feedback. The use of the package to change attitudes to vaccination was also investigated (Amos et al., 2018). The resource is available for free download online (www.simfection.org.uk ) to allow access to interested parties. The website allows users to enter their email address, which will allow further evaluation of the educational resource to be completed at a later date.

Acknowledgements

The authors would like to thank the Society for Applied Microbiology for contributing to the support of the SimFection project through its public engagement grants. The author would also like to thank the Faculty of Science and Engineering at Manchester Metropolitan University for supporting this research project through its studentship programme.

References

Carolan, K., J.Verran, M.Crossley, J.Redfern, N.Whitton, M.Amos. 2018. Impact of educational interventions on adolescent attitudes and knowledge regarding vaccination: A pilot study. PLoS ONE 13(1): e0190984.

AQA. 2014. GCE AS and A Level Specification: Biology AQA

Beigel, J.H., J. Farrar, A.M. Han, F.G. Hayden, R, Hyer, M.D. De Jong, S. Lochindarat, N.T.K. Tien, N.T. Hien, and T.T. Hien. 2005. Avian Influenza A (H5N1) Infection in Humans. New England Journal of Medicine 353 (13): 1374-1385. 
Crossley, M., and M. Amos. 2011. SimZombie: A Case-Study in Agent-Based Simulation Construction. 'Agent and Multi-Agent Systems, Technologies and Applications: Lecture notes in Artificial Intelligence' vol 6682, Edited by J.O’Shea, N.T.Nguyen, K.Crockett, R.J.Howlett and L.C.Jain, 514 - 523. Berlin, Heidelberg. Springer.

Donaldson, L. J., P. D. Ellis, B. M. Greaves, F.E.C. Mytton, O. T.Peabody, R. G. Yardley, and I.E. Rutter. 2009. Mortality from Pandemic A/H1N1 2009 Influenza in England: Public Health Surveillance Study. British Medical Journal 339 b5213.

Dube, E., D. Gagnon, and N. MacDonald. 2015. Strategies Intended to Address Vaccine Hesitancy: Review of Published Reviews. Vaccine, 33:1-11.

Fenner, Frank. 2011. “Smallpox Eradication: The Vindication of Jenner's Prophesy.” In History of Vaccine Development, 27-32. Berlin, Heidelberg. Springer.

Fraser, C., C. A. Donnelly, S. Cauchemez, W. P. Hanage, M. D. Van Kerkhove, T. D. Hollingsworth, J. Griffin, et al. 2009. Pandemic Potential of a Strain of Influenza A (H1N1): Early Findings. Science 324 (5934): 1557-1561.

Gowda, C., S. E. Schaffer, K. J. Dombkowski, and A. F. Dempsey. 2012. Understanding Attitudes toward Adolescent Vaccination and the Decision-Making Dynamic among Adolescents, Parents and Providers. BMC Public Health 12 (1). 509.

Gupta, R. K., J. Best, and E. MacMahon. 2005. Clinical Review: Mumps and the UK Epidemic 2005. British Medical Journal 330 (May): 1132-1135.

Jansen, V, N. Stollenwerk, H. J.Jensen, M. E. Ramsay, W. J. Edmunds, and C. J. Rhodes. 2003. Measles Outbreaks in a Population with Declining Vaccine Uptake. Science 301 (5634): 804.

Kay, D., M. Roche, J. Atkinson, K. Lamden, and R. Vivancos. 2011. Mumps Outbreaks in Four Universities in the North West of England: Prevention, Detection and Response. Vaccine 29 (22): 3883-3887. 
Li, K. S., Y. Guan, J. Wang, and G. J. D. Smith. 2004. Genesis of a Highly Pathogenic and Potentially Pandemic H5N1 Influenza Virus in Eastern Asia. Nature 430 (6996): 209 - 213. Nyhan, B., J. Reifler, S. Richey, and G. L. Freed. 2014. Effective Messages in Vaccine Promotion: A Randomized Trial. Pediatrics, 133 (4), e835-e842.

OCR. 2013. AS/A Level GCE Biology Specification. OCR

Ramsay, M. E. 2013. Measles: The Legacy of Low Vaccine Coverage. Archives of Disease in Childhood 98 (10): 752-754.

Selwyn, N., and R. Cooper. 2015. "The Potential of Digital Technology for Science Learning and Teaching-The Learners' Perspective.” In The Future in Learning Science: What's in It for the Learner? pp 263-277. Berlin, Heidelberg. Springer.

Subbarao, K., and T. Joseph. 2007. Scientific Barriers to Developing Vaccines against Avian Influenza Viruses. Nature Reviews Immunology 7: 267-278.

Virus, Novel Swine-Origin Influenza A H1N1, and Investigation Team. 2009. "Emergence of a Novel Swine-Origin Influenza A (H1N1) Virus in Humans.” New England Journal of Medicine 2009 (360): 2605-2615.

Verran J., M. Crossley, K.Carolan, N.Jacobs and M. Amos. 2014. Monsters, microbiology and mathematics: the epidemiology of a zombie apocalypse. Journal of Biological Education 48 (2): $98-104$.

WJEC. 2010. GCE Examinations from 2009: Biology. WJEC

Yang, Y., M E. Halloran, J. D. Sugimoto, and I. M. Longini. 2007. Detecting Human-toHuman Transmission of Avian Influenza A ( H5N1 ). Emerging Infectious Diseases 13 (9).1969- 1971

Zimmerman, Eric. 2014. “The Iterative Design Process.” In Game Design Workshop: A Playcentric Approach to Creating Innovative Games, edited by T.Fullerton. New York. CRC press. 
List of tables:

Table 1: Data used to set the parameters for each infectious disease simulation

List of Supplementary Information

Supplement 1: Feedback form issued to students

Supplement 2: Feedback form issued to teachers

Supplement 3: Changes suggested to SimFection by teachers in January $2015(\mathrm{n}=12)$, and the responses to those suggestions.

Supplement 4: Changes suggested to SimFection by teachers in April $2015(n-=20)$, and the responses to those suggestions.

List of figures:

Figure 1.Screenshots from SimFection showing representative images of different simulations. The circles represent individuals who are immunised (light blue), symptomatic (red outline, dark red centre), healthy (black outline), recovered (red outline, white centre), infected (red outline, yellow centre) or deceased (grey): a) measles, demonstrating how infectious diseases can spread quickly when vaccination coverage is not sufficient; the simulation shown simulates how quickly measles would spread if vaccination coverage is low;b) smallpox, to demonstrate how some infectious diseases can be contained and eradicated using ring vaccination. Students 'click' to vaccinate a ring of individuals around infected individuals to attempt to contain the spread of infection; c) influenza to demonstrate how different mortality and infectivity rates affect the spread of infectious disease. On the left simulation, low mortality rate and high infectivity rate is used to simulate H1N1 (avian influenza), causing the infection to spread quickly but not kill many people. On the right simulation, high mortality rate and low infectivity is used to simulate H5N1 (swine 
influenza), resulting in an infection that kills most infected individuals but does not spread throughout the population quickly as it kills people before they can pass on the infection to many people; d) mumps, to demonstrate how outbreaks can occur when unimmunised populations previously protected through 'herd immunity' move from areas with high vaccination coverage (left side of screen) to areas with low vaccination coverage (right side of screen).

Figure 2. Stages of development of the SimFection learning package

Figure 3. Responses of teachers before and after improvements to the resource $(n=12 ; n=20)$

Figure 4. Responses of students before and after improvements to the resource $(n=36 ; n=20)$

Table 1. Data and sources of information used to set the parameters for each infectious disease simulation. Key websites used were: www.cdc.gov, www.nhs.uk, www.flu.gov and www.who.int. 
$* \mathrm{R}_{0}=$ basic reproduction number. This is the number of cases generated by one case of an infectious

\begin{tabular}{|c|c|c|c|c|c|}
\hline & Measles & Mumps & $\begin{array}{l}\text { Influenza } \\
\text { (H1N1) }\end{array}$ & $\begin{array}{l}\text { Influenza } \\
\text { (H5N1) }\end{array}$ & Smallpox \\
\hline $\begin{array}{l}\text { Incubation } \\
\text { period } \\
\text { (average) }\end{array}$ & $\begin{array}{ll}10 & \text { days } \\
\text { (NHS) } & \end{array}$ & 17 days (NHS) & 2 days (CDC) & $\begin{array}{l}8 \text { days(Beigel } \\
\text { et al. 2005) }\end{array}$ & 12 days $(C D C)$ \\
\hline $\begin{array}{l}\text { Infectious } \\
\text { period }\end{array}$ & 9 days (NHS) & 7 days (CDC) & 7 days (CDC) & 10 days (CDC) & 20 days (CDC) \\
\hline $\begin{array}{l}\text { Length of } \\
\text { symptoms }\end{array}$ & $\begin{array}{ll}10 & \text { days } \\
(\mathrm{NHS}) & \end{array}$ & 10 days (CDC) & $\begin{array}{ll}3-4 & \text { days } \\
(\mathrm{CDC}) & \end{array}$ & 10 days (CDC) & 20 days (CDC) \\
\hline $\begin{array}{l}\text { Case-fatality } \\
\text { rate }\end{array}$ & $0.2 \%(C D C)$ & $0.01 \%$ (WHO) & $\begin{array}{l}0.026 \% \\
\text { (Donaldson } \\
\text { et al. 2009) }\end{array}$ & $60 \%$ (Flu.gov) & $30 \%(C D C)$ \\
\hline $\begin{array}{l}\text { Infectivity ( } \mathrm{R}_{0} \\
\text { value*) }\end{array}$ & $18(C D C)$ & $7(\mathrm{CDC})$ & $\begin{array}{ll}1.6 & \text { (Fraser } \\
\text { et al. 2009) }\end{array}$ & $\begin{array}{l}0.01 \%^{\dagger} \\
\text { (Yang et al. } \\
2007)\end{array}$ & $7(\mathrm{CDC})$ \\
\hline
\end{tabular}

disease over the infectious period. ${ }^{\dagger}$ Human to human transmission of H5N1 is very low and so a percentage chance of transmission is given rather than a $\mathrm{R}_{0}$ number. 
Figure 1.Screenshots from SimFection showing representative images of different simulations. The circles represent individuals who are immunised (light blue), symptomatic (red outline, dark red centre), healthy (black outline), recovered (red outline, white centre), infected (red outline, yellow centre) or deceased (grey): a) measles, demonstrating how infectious diseases can spread quickly when vaccination coverage is not sufficient; the simulation shown simulates how quickly measles would spread if vaccination coverage is low; b) smallpox, to demonstrate how some infectious diseases can be contained and eradicated using ring vaccination. Students 'click' to vaccinate a ring of individuals around infected individuals to attempt to contain the spread of infection; c) influenza to demonstrate how different mortality and infectivity rates affect the spread of infectious disease. On the left simulation, low mortality rate and high infectivity rate is used to simulate H1N1 (avian influenza), causing the infection to spread quickly but not kill many people. On the right simulation, high mortality rate and low infectivity is used to simulate H5N1 (swine influenza), resulting in an infection that kills most infected individuals but does not spread throughout the population quickly as it kills people before they can pass on the infection to many people; d) mumps, to demonstrate how outbreaks can occur when unimmunised populations previously protected through 'herd immunity' move from areas with high vaccination coverage (left side of screen) to areas with low vaccination coverage (right side of screen). 


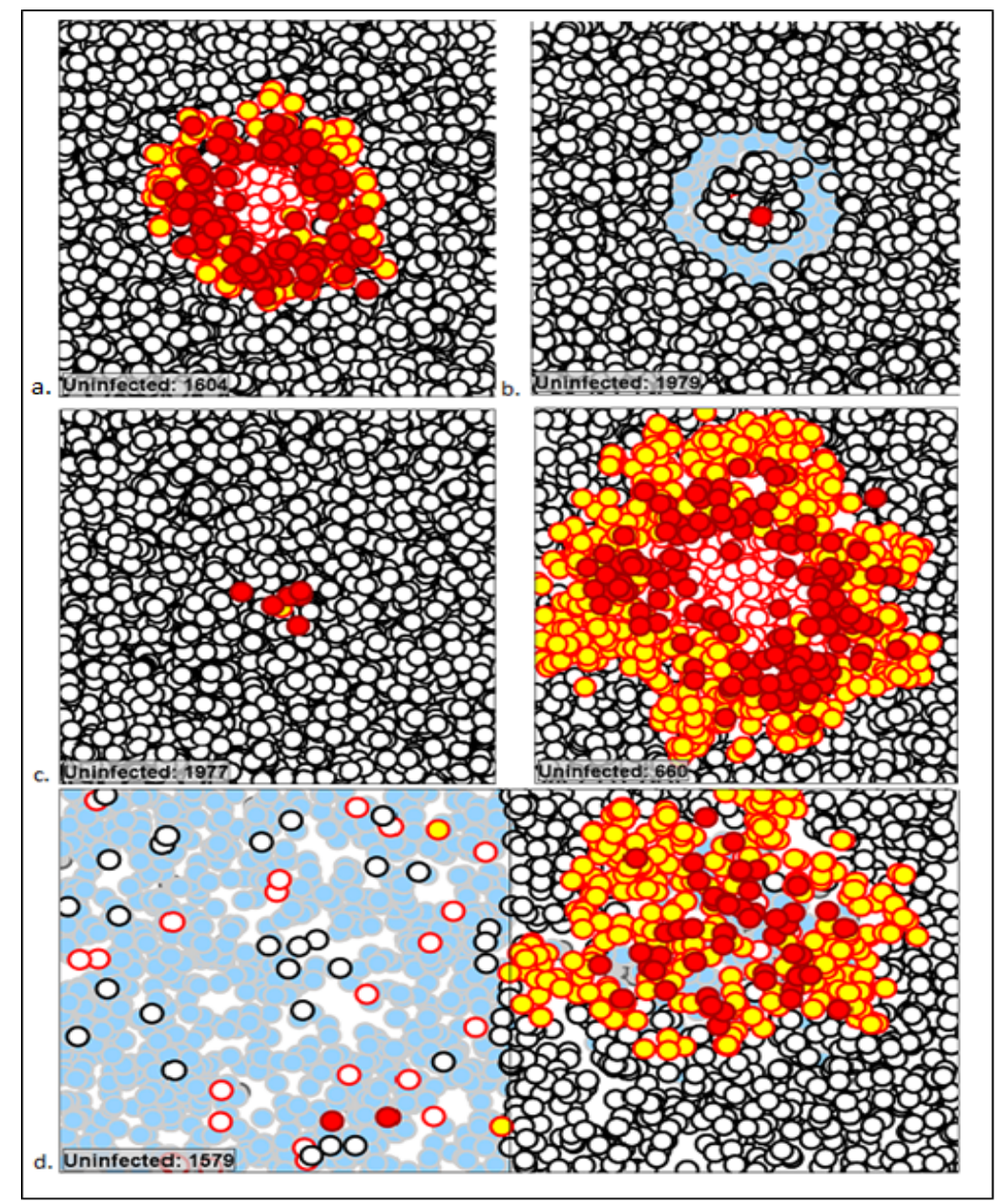


Figure 2. Stages of development of the SimFection learning package

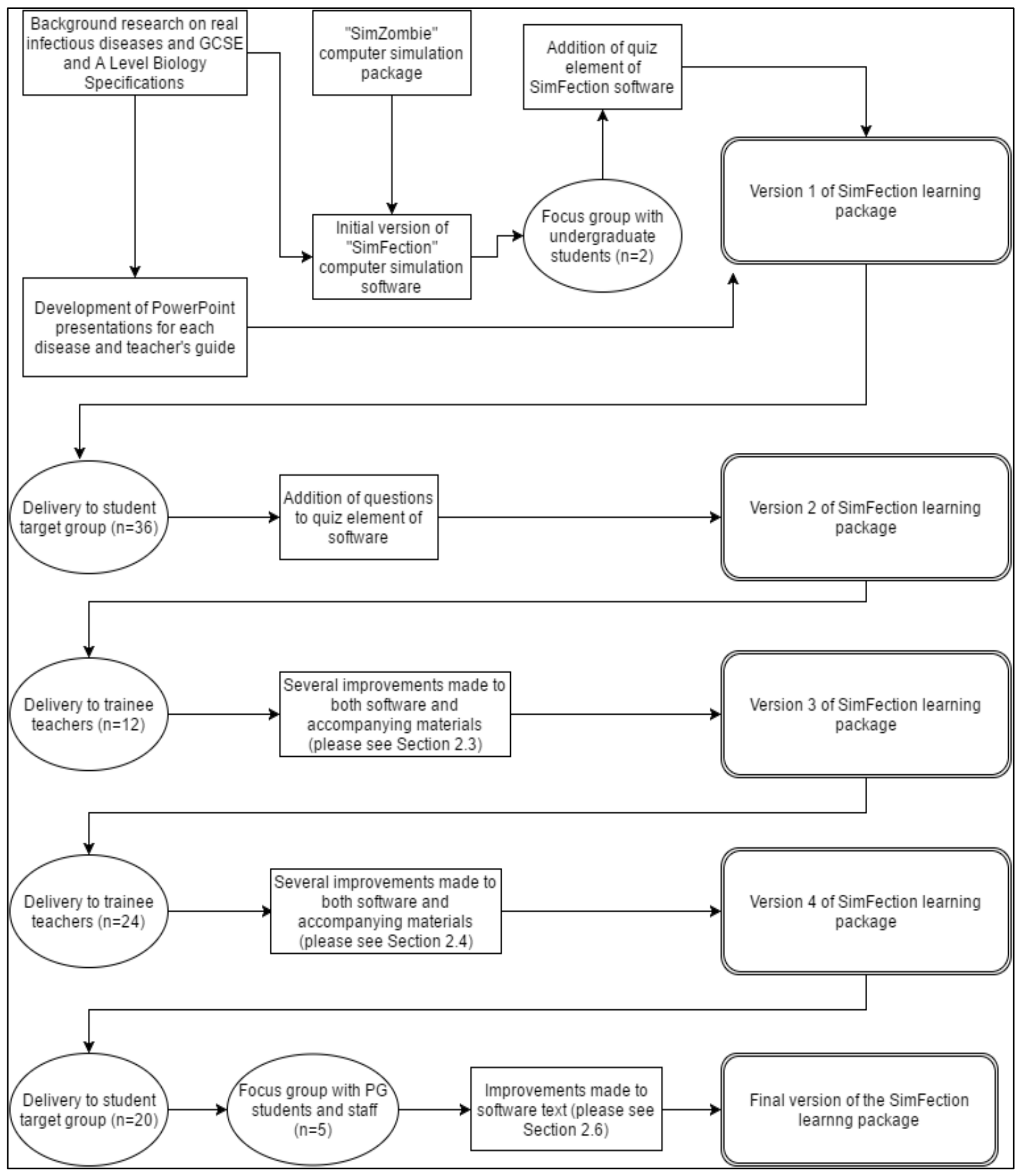


Figure 3. Responses of teachers before and after improvements to the resource

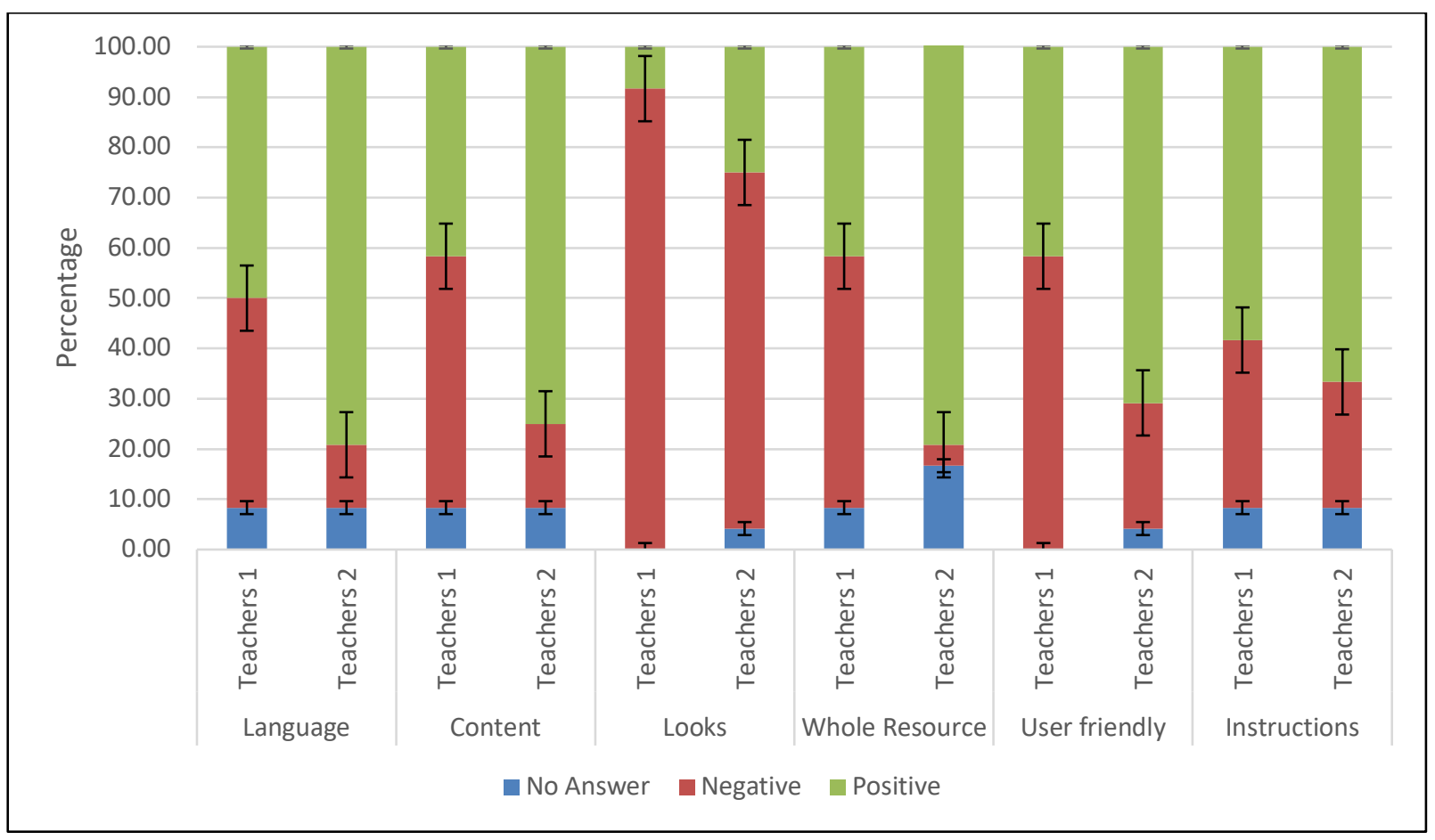

Figure 4. Responses of students before and after improvements to the resource 


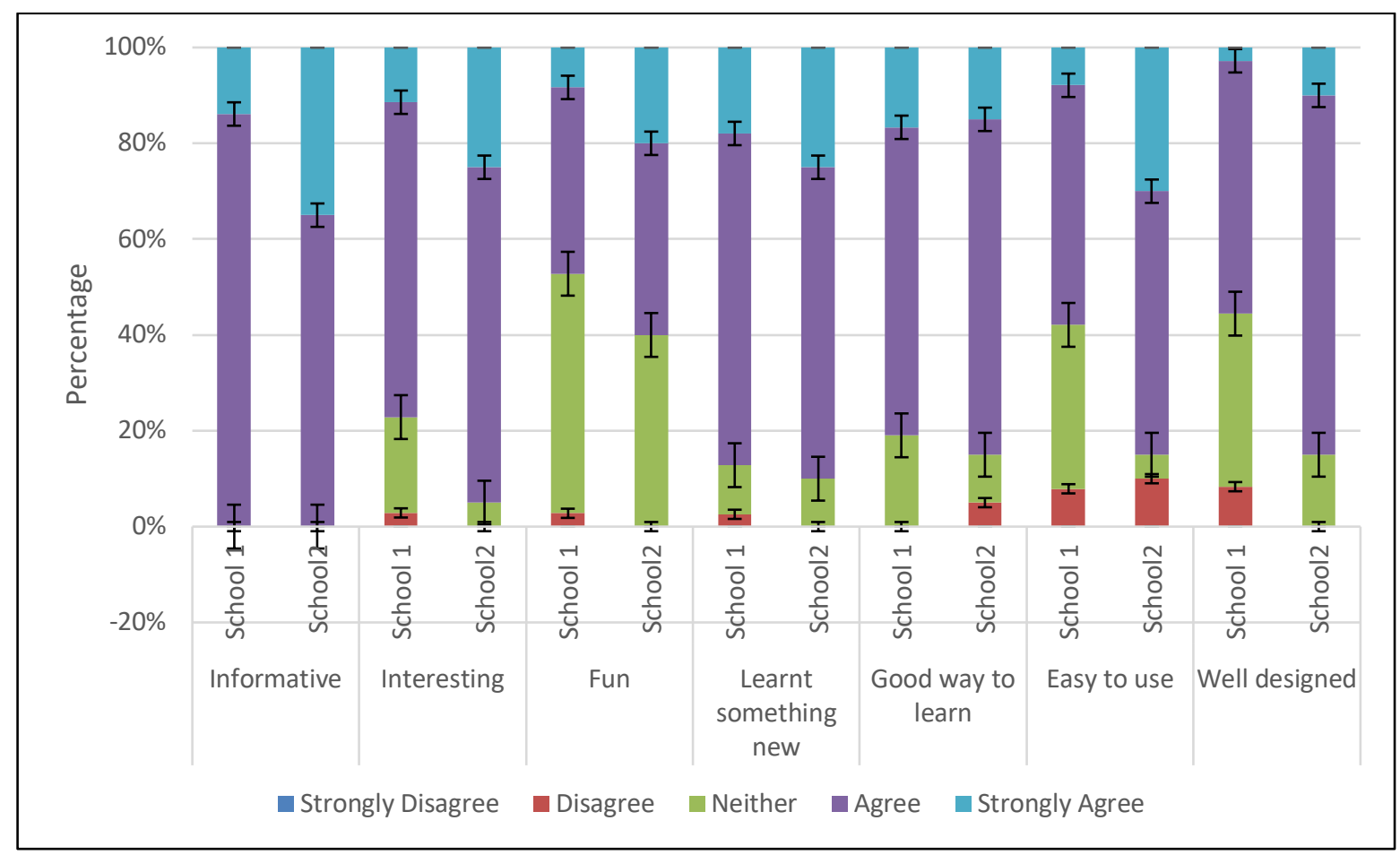

